\title{
Effect of Wearing the Elevation Training Mask on Physiological Performance in Elite Kayaking Girls
}

Amine Ghram ${ }^{1,2,3}$, Farahnaz Amirshaghaghi ${ }^{1 *}$, Nicola Bragazzi ${ }^{4}$, Hesam Addin Akbari ${ }^{1}$, Hamdi Chtourou ${ }^{5}$, Carl J Lavie ${ }^{6}$, David Jiménez-Pavón ${ }^{7,8,9}$

${ }^{1}$ Department of Physical Education and Sports Science, Faculty of Physical Education, University of Tehran, Tehran, Iran.

${ }^{2}$ Healthy Living for Pandemic Event Protection (HL - PIVOT) Network, Chicago, IL, USA

${ }^{3}$ Department of Cardiac Rehabilitation, Tehran Heart Center, Tehran University of Medical Sciences, Tehran, Iran.

${ }^{4}$ Centre for Disease Modelling, York University, Toronto M3J 1P3, Canada

${ }^{5}$ LR19JS01 EM2S: Éducation, Motricité, Sport et Santé, High Institute of Sport and Physical Education of Sfax, University of Sfax, Tunisia

${ }^{6}$ Department of Cardiovascular Diseases, John Ochsner Heart and Vascular Institute, Ochsner Clinical School the University of Queensland School of Medicine, New Orleans, Louisiana, USA

${ }^{7}$ MOVE-IT Research Group, Department of Physical Education, Faculty of Education Sciences, Universidad de Cádiz, Spain

${ }^{8}$ Biomedical Research and Innovation Institute of Cádiz (INiBICA) Research Unit, Puerta del Mar University Hospital, University of Cádiz, Spain

${ }^{9}$ CIBER of Frailty and Healthy Aging (CIBERFES), Madrid, Spain. 
Amine Ghram: Amineghram.110@ut.ac.ir

Farahnaz Amirshaghaghi: famirshaghaghi@ut.ac.ir

Nicola Bragazzi: robertobragazzi@gmail.com

Hesam Addin Akbari: hesameakbary@ut.ac.ir

Hamdi Chtourou: $\underline{\text { h_chtourou@yahoo.fr }}$

Carl J Lavie: clavie@ ochsner.org

David Jiménez-Pavón: david.jimenez@uca.es

- Correspondence concerning this article should be addressed to Farahnaz Amirshaghaghi: Department of Physical Education and Sports Science, Faculty of Physical Education, University of Tehran, Tehran, Iran.

E-mail: famirshaghaghi@ut.ac.ir

- Nicola Luigi Bragazzi, MD, PhD, MPH is the submitted author on behalf of other authors.

Centre for Disease Modelling, York University, Toronto M3J 1P3, Canada

E-mail: robertobragazzi@gmail.com

Tel: 1 (416) 736 5243; Fax: 1 (416) 7365698 


\title{
Effect of Wearing the Elevation Training Mask on Physiological Performance in Elite Kayaking Girls
}

\begin{abstract}
Introduction: The Elevation Training Mask 2.0 (ETM) has been introduced as a novel tool to allow for respiratory muscle training and altitude exposure during exercise that can improve performance and hematological markers in elite and well-trained athletes. Thus, the aim of the present study was to assess the effect of wearing ETM while training on erythropoietin (EPO), vascular endothelial growth factor (VEGF), peak oxygen consumption $\left(\mathrm{VO}_{2 \mathrm{max}}\right)$, lactate, and 1000 m kayaking performance in elite Kayaking girls.
\end{abstract}

Methods: Thirty elite Kayaking girls (14 to 18-year-old) were equally and randomly assigned into mask, no mask, and control groups. The mask and no-mask groups completed 8 weeks of repeated sprint training, while the control group performed their routine kayaking training programs. Preand post-training tests included EPO, VEGF, $\mathrm{VO}_{2 \max }$, lactate, and 1000-m kayaking performance.

Results: $1000-\mathrm{m}$ time trial significantly decreased for mask $(\mathrm{p}<0.001)$ and control $(\mathrm{p}=0.035)$ groups, and was significantly lower in mask group than control $(\mathrm{p}=0.014)$ and no mask $(\mathrm{p}=0.009)$ groups. EPO did not show any significant changes for all groups. VEGF was increased significantly for mask $(p=0.04)$ and no mask $(p=0.014)$ groups. Lactate was decreased significantly for mask group $(\mathrm{p}=0.025) . \mathrm{VO}_{2 \max }$ increased significantly for no mask group $(\mathrm{p}=0.021)$.

Conclusion: Wearing the ETM while participating in 8 weeks of repeated sprint training might improve specific blood markers and endurance performance, especially anaerobic pathways. Performing repeated sprint training while wearing ETM has the potential to enhance performance in Kayak racing. 
Keywords: Airflow restriction, Physical exercise, Respiratory Muscle Training, Endurance performance, VEGF, EPO. 


\section{Introduction}

Flat-water Kayaking, as a high intensity endurance event, requires development of both aerobic and anaerobic capacities. Respiratory muscle training (RMT) has become a popular training method among competitive endurance athletes to improve athletic performance outcomes (Granados, Gillum, Castillo, Christmas, \& Kuennen, 2016; Porcari et al., 2016). RMT can improve cycling endurance (Markov, Spengler, Knöpfli-Lenzin, Stuessi, \& Boutellier, 2001), decrease inspiratory muscle fatigue, and improve time trial performance (Romer, McConnell, \& Jones, 2002).

The Elevation Training Mask v2.0 (ETM) is a commercially manufactured (Training Mask LLC; Cadillac, MI, USA) RMT device that uses a silicone mask and flexible neoprene head strap to seal the face during exercise (Granados et al., 2016). The adjustable "resistance caps" on this device also purportedly provide for between 3,000 and 18,000 ft of "Altitude Resistance" (Granados et al., 2016). Wearing the ETM acts as a respiratory training device,allows the user to simulate altitudes ranging from $914 \mathrm{~m}$ to $5486 \mathrm{~m}$ and is used by high performance athletes to simulate the increased expiratory and inspiratory effort required at high elevation (Porcari et al., 2016)

It was shown that wearing ETM alone did not improve lung function, inspiratory muscle strength, or stimulate changes in hemoglobin or hematocrit levels (Porcari et al., 2016). However, wearing ETM while participating in a 6-week high-intensity cycle ergometer interval training program may improve physical capacity and endurance performance in moderately trained students (Porcari et al., 2016). Combining breathing resistance with exercise training can improve $\mathrm{VO}_{2 \text { peak }}$ (Kido et al., 2013). We can speculate that wearing ETM while participating in repeated sprint training may benefit endurance athletes by means of specific adaptations in Kayakers. As well, we have hypothesized the role of the hormone erythropoietin (EPO) and vascular endothelial growth factor 
(VEGF) which are the important factors regulating erythropoiesis and angiogenesis (Ke \& Costa, 2006; Lee, Bae, Jeong, Kim, \& Kim, 2004; Wiśniewska, Płoszczyca, \& Czuba, 2020) , respectively, and lactate concetration as an indicator of respiratory muscle performance.

The physiological adaptations that occur during EMT depend on the specific training protocol. However, no study has reported the effects of wearing EMT while exercising on physiological Kayaking performance. It would be of interest to know if wearing ETM would further enhance physiological kayaking performance and by which specific adaptation. Therefore, the purpose of the present study was to investigate the changes of blood EPO and VEGF, lactate levels, $\mathrm{VO}_{2 \max }$, and endurance performance in elite Kayaking girls.

\section{Methods}

\subsection{Participants and study design}

Thirty female national level Sprint Kayak athletes (mean \pm SD: age: $14 \pm 3$ years; height: $1.62 \pm$ $0.67 \mathrm{~m}$; body mass: $50.7 \pm 4.8 \mathrm{~kg}$ ) were recruited for this study. The participants had at least 2 years of kayak specific training experience, trained regularly 5 training sessions of $2 \mathrm{~h}$ per week, had been training regularly with a Kayak ergometer, and had to be competing at national level. The study took place during the competitive season. They did not have any medical conditions and were free of acute or chronic injuries over the 6 months before the experiment. All of the participants reported that they had not climbed more than 2500 meters in the 6 months prior to the study. Parents

and coaches of all participants were asked to give their written informed consent following a detailed explanation about the aims, benefits, and potential risks involved in the investigation. The study was approved by the university ethics review board and human subjects committee with ethics No: IR.UT.SPORT.1399.018. 
The participants were randomly and equally assigned into three groups (10 participants each): Mask group, no mask group, and a control group. The mask group performed repeated sprint training while wearing ETM according to ETM manufacturer settings during all training sessions (Porcari et al., 2016). The mask was set to simulate an altitude of $914 \mathrm{~m}$ (week 1), $1829 \mathrm{~m}$ (week 2), $2743 \mathrm{~m}$ (week 3 and 4), $3658 \mathrm{~m}$ (week 5, 6, 7, and 8) (Porcari et al., 2016). The no mask group performed repeated sprint training without wearing ETM. The control group performed their normal training program without wearing ETM.

Within 10 days after completion the training protocol, all participants performed the exercise test with the same protocol but under normal conditions. Before each testing session, all athletes performed a standard warm up that consisted of 3-min paddling at $\sim 85 \%$ maximum Heart Rate $\left(\mathrm{HR}_{\max }\right)$ followed by two $15 \mathrm{~s}$ accelerations interspersed with $45 \mathrm{~s}$ rest and two standing starts of 24 strokes with 45 s rest between each ending with 3 min of paddling at $85 \% \mathrm{HR}_{\max }$. Similarly, following each session the athletes also completed a standard cool down consisting of 5 min of paddling at $75 \% \mathrm{HR}_{\max }$. The main body of each of sprint kayak training sessions consisted of repeated sprint training.

\subsection{VO2max Test}

The participants performed an incremental test in normoxia condition using friction loaded cycle ergometer (Monark 894 ${ }^{\mathrm{E}}$; Stockholm, Sweden) interfaced with a microcomputer in order to measure $\mathrm{VO}_{2 \max }$. The incremental exercise test began with a load of $40 \mathrm{~W}$, with increments of 25 W every 2 minutes. $\mathrm{VO}_{2}$ was continuously recorded during the test and the test was continued to exhaustion or until the participant was unable to maintain the minimal cadence of $60 \mathrm{rpm}$. Finally, $\mathrm{VO}_{2 \max }$ was indirectly calculated based on the prediction equation determined by Rocha et al. 
(Rocha, Louro, Matias, Brito, \& Costa, 2016). HR (Polar A1, Polar Electro, Kempele, Finland) was measured continuously throughout the test.

\subsection{Time Trial test}

Kayak athletes were familiar with the use of ergometer and the 1000-m kayaking time trial protocol to assess rowing performance. They performed the graded exercise test according to the study of Kerr et al. (Kerr, Spinks, Leicht, Sinclair, \& Woodside, 2008) on a rowing ergometer (Concept IID, Morrisville, USA). The warm-up was followed by a 1000-m effort with the participants encouraged to adopt a self-selected stroke rate and to maximize force on the ergometer paddle to complete the 1000-m in the fastest time (Kerr et al., 2008). All kayak athletes started this test at the same time and their time was recorded.

\subsection{Blood Analysis}

Venous blood samples $(10 \mathrm{ml})$ were collected by venipuncture of the antecubital vein into serum separator or EDTA-coated tubes before and after the exercise training protocol. Plasma and serum samples were aliquoted and stored at $-80^{\circ} \mathrm{C}$ until analysis. The biomarkers were measured by enzyme-linked immunosorbent assay kits according to the manufacturer's instructions: serum EPO (IBL international GmbH, Hamburg, Germany) and plasma VEGF (IBL international GmbH, Hamburg, Germany). Lactate was measured using the Lactate Pro 2 Analyzer (Arkray, Tokyo, Japan).

\subsection{Statistical Analysis}

The commercial software Statistical Package for the Social Sciences (SPSS) for Windows, version 25.0 (SPSS Inc., Chicago, IL, USA) was utilized for all statistical analyses. Results are presented as means \pm standard deviation (SD). Data normality was tested using Shapiro-Wilk test. Mean 
values were compared using Student's t-test. Comparison among groups was performed by twoway ANOVA with Fisher's Least Significant Difference (LSD) test as post-hoc test. The significance level was established at $\mathrm{p}<0.05$.

\section{Results}

As shown in Table 1, 1000-m kayaking record was significantly decreased for the mask group (p $<0.001)$. EPO did not show any significant changes for all groups. VEGF was increased significantly for the mask $(\mathrm{p}=0.04)$ and for the no-mask $(\mathrm{p}=0.014)$ groups. Lactate was decreased significantly for the mask group $(\mathrm{p}=0.025)$. $\mathrm{VO}_{2 \max }$ increased significantly for the no-mask group $(\mathrm{p}=0.021)$

Two-way ANOVA test showed a significant interaction for only 1000-m kayaking record $(\mathrm{p}=$ 0.014). LSD post-hoc test showed that after the exercise training protocol, 1000-m kayaking record was significantly lower in mask group than control $(\mathrm{p}=0.014)$ and no-mask $(\mathrm{p}=0.009)$ groups, respectively.

\section{Discussion}

The present study investigated the effects of wearing ETM while participating in repeating sprint training on EPO, VEGF, lactate, $\mathrm{VO}_{2 \max }$, and 1000-m kayaking record in the elite Kayaking girls. The main findings of this study were that in Kayak athletes who trained with or without ETM device, VEGF levels increased and EPO level showed no change. Training with ETM improved 1000-m kayaking record, and decreased lactate levels, while training without ETM increased $\mathrm{VO}_{2 \max }$.

To the best of our knowledge, no previous study was investigated the effects of RMT on EPO, VEGF and performance parameters simultaneously. Therefore, this makes direct 
comparisons to previous literature more difficult. We compared our results with the findings of previous studies that examined the effect of altitude training or simulated altitude training (hypoxia training). Our results indicate that the increase of VEGF depends of the training method adopted which is in line with previous studies. Similar to previous study (Wahl et al., 2013), authors showed that exercise is effective in VEGF stimulation when it is combined with hypoxia. VEGF also increased during intermittent hypoxic training (Wiśniewska et al., 2020). Asano et al. (Asano et al., 1998) showed that serum VEGF levels increased in swimmers after 3 weeks of endurance training at moderate terrestrial altitude $(1886 \mathrm{~m})$. Results obtained by Park and Lim (Park \& Lim, 2017) indicated a rise of serum VEGF in swimmers following 6 weeks of a combination of continuous, interval and resistance training in hypobaric hypoxic conditions (in a simulated altitude of $3000 \mathrm{~m}$ ). On the contrary, in groups which trained in normoxia, there were no changes in VEGFA level (Wiśniewska et al., 2020). Thus, both repeated sprint training and hypoxic training could stimulate VEGF improvements explaining our results.

On the other hand, the present study showed that EPO did not change significantly after training with and without ETM, which is similar to a previous study where EPO did not change during "intermittent hypoxic training" (Wiśniewska et al., 2020). However, EPO increased after "live high - base train high - interval train low" and "live high - train low" conditions (Wiśniewska et al., 2020) and following several hours of hypoxic exposure (González et al., 2006; Mackenzie, Watt, \& Maxwell, 2008). The lack of EPO response to hypoxic and normoxic training can be associated with the inefficiency of hypoxic training with ETM to elicit hematological changes, which was confirmed in cyclists and swimmers (Czuba, Fidos-Czuba, Płoszczyca, Zając, \& Langfort, 2018; Czuba et al., 2011). Thus, longer periods of exposure to hypoxia are likely needed to obtain an increase in EPO levels, probably, being the best option the concept of "live high-train low". 
Thereby, training with ETM was effective for VEGF increment and ineffective for EPO increment. Therefore, our finding indicated that ETM did not induce hypoxia and the Kayaking athletes should perform new respiratory training methods that induce a sufficient hypoxic dose.

We also reported a significant reduction in lactate levels in the mask group. In line with the present data, Jagim et al. (Jagim et al., 2018)showed that blood lactate values after sprint during the ETM condition was lower as compared without ETM condition in recreational weight lifters (Jagim et al., 2018). On the other hand, previous studies reported no significant changes in lactate after continuous exercise with mask and continuous exercise without mask (Barbieri et al., 2020), 2, 4, and 6 weeks of high intensity cycle ergometer training using ETM (Porcari et al., 2016), and 6 weeks of training with ETM, and intermittent hypoxic training (IHT) (Bellovary et al., 2019).

These results partially confirm our finding in lower lactate levels after repeated sprint kayak training. However, the differences between the results of these studies may be due to the different training protocols and sport modalities, as well as to background of anaerobic training.

The increase of $\mathrm{VO}_{2 m a x}$ in no-mask group indicates that repeated training exercise is able to enhance exercise capacity. Repeated sprint with higher maximal aerobic power and $\mathrm{VO}_{2 m a x}$ are positively related to $1000-\mathrm{m}$ time trial performance. Additionally, it has been demonstrated that physiological characteristics, such as higher maximal aerobic power and $\mathrm{VO}_{2 \mathrm{max}}$, are better related to $1000-\mathrm{m}$ time-trial performance (Borges, Dascombe, Bullock, \& Coutts, 2015).

Training without ETM increased $\mathrm{VO}_{2 \max }$ that indicate that ETM did not play an important in improving exercise capacity by means of $\mathrm{VO}_{2 \max }$ and did not act like a hypoxia stimulator. Thus, $\mathrm{VO}_{2 \max }$ is affected by the type of training, not the ETM2 condition. This finding is in line with Biggs et al. (Biggs, England, Turcotte, Cook, \& Williams, 2017) who showed that high intensity 
interval training is a viable method to increase $\mathrm{VO}_{2 \max }$, but ETM may not lead to greater overall improvements. Also, Porcari et al. (Porcari et al., 2016) in moderately trained participants showed that 6 weeks of high intensity cycle ergometer training using ETM did not improve $\mathrm{VO}_{2 \max }$ more than did the control condition. To highlight is the fact that the current study did not found increase in $\mathrm{VO}_{2 \max }$ using a non-sport specific test (cycle ergometer) while the sport specific performance (time in 1000-m kayaking) was improved. However, research has indicated that simulated altitude should be 2000 to 3000 meters to provide obvious changes in physiological changes and increased aerobic performance (George, Vehrs, Allsen, Fellingham, \& Fisher, 1993). Although the simulated altitude in the present study was ranged from $914 \mathrm{~m}$ to $3,658 \mathrm{~m}$, factors other than altitude, such as training with ETM duration, may also play an important role in the conflict between studies.

The training protocol used in the present study was repeated sprint training which is a potential useful tool for monitoring training in Sprint Kayak athletes. Such information may be used to guide coaches and sports scientists on appropriate programs to develop elite kayak athletes in each of the specific Sprint Kayak events. Such data may help to inform Sprint Kayak coaches and coaches in similar sports involving aerobic and anaerobic metabolism when designing appropriate targeted training sessions that meet the specific physical and physiological aspects for individual athletes or events.

We also showed that training without mask, by increasing VEGF and $\mathrm{VO}_{2 \max }$, did not improve endurance performance. These data in addition to the mask condition data implies that lactate levels (clearance) could be more determinant than $\mathrm{VO}_{2 \max }$ and VEGF levels in predicting endurance performance. Our finding is in line with those of Bassett et al. (Bassett \& Howley, 2000), which had shown that the speed of lactate threshold is the best physiological predictor of endurance performance. 
On the other hand, lack of positive effects of ETM on $\mathrm{VO}_{2 \max }$ and EPO may be due that the mask can cause some psychological discomfort, exercise intolerance, and inadequate hyperventilation that led to arterial hypoxemia (Granados et al., 2016).

A strength of the present study that we included a no-mask group that trained without mask and a control group that continued his training routine. A limitation of the present study is the insufficient duration for ETM exposure. Future studies should increase the simulated elevation and should compared this device with other approaches.

\section{Conclusion}

Wearing ETM while training may be effective in increasing VEGF, decreasing lactate, and improving anaerobic endurance performance of elite female kayaking athletes. Furthermore, using ETM inhibited increases in $\mathrm{VO}_{2 \max }$ and EPO. These findings should be interpreted with caution. Training without ETM is able to improve $\mathrm{VO}_{2 \max }$ but not performance. This study confirms that ETM is not a stimulator of hypoxia but could be useful to promote anaerobic adaptations. Future researches should continue investigating the exercise adaptations when using ETM.

\section{Acknowledgments}

The authors would like to thank all participants for their time and commitment in undertaking this study.

Thanks to Biomedical Research Networking Center on Frailty and Healthy Aging (CIBERFES) and FEDER funds from the European Union (CB16/10/00477),

\section{Institutional Review Board Statement}

The study was conducted according to the guidelines of the Declaration of Helsinki, and approved by the Institutional Review Board of the University of Tehran. 


\section{Informed Consent Statement}

Informed consent was obtained from all subjects involved in the study.

\section{Conflicts of Interest}

The authors declare no conflict of interest.

\section{References}

Asano, M., Kaneoka, K., Nomura, T., Asano, K., Sone, H., Tsurumaru, K., . . Okuda, Y. (1998). Increase in serum vascular endothelial growth factor levels during altitude training. Acta Physiol Scand, 162(4), 455-459. doi:10.1046/j.1365-201X.1998.0318e.x

Barbieri, J. F., Gáspari, A. F., Teodoro, C. L., Motta, L., Castaño, L. A. A., Bertuzzi, R., . . de Moraes, A. C. (2020). The effect of an airflow restriction mask (ARM) on metabolic, ventilatory, and electromyographic responses to continuous cycling exercise. PLoS One, 15(8), e0237010. doi:10.1371/journal.pone.0237010

Bassett, D. R., Jr., \& Howley, E. T. (2000). Limiting factors for maximum oxygen uptake and determinants of endurance performance. Med Sci Sports Exerc, 32(1), 70-84. doi:10.1097/00005768200001000-00012

Bellovary, B. N., King, K. E., Nuñez, T. P., McCormick, J. J., Wells, A. D., Bourbeau, K. C., . . Mermier, C. M. (2019). Effects of high-intensity interval training while using a breathing-restrictive mask compared to intermittent hypobaric hypoxia. Journal of Human Sport and Exercise, 14(4), 13. doi:10.14198/jhse.2019.144.11

Biggs, N. C., England, B. S., Turcotte, N. J., Cook, M. R., \& Williams, A. L. (2017). Effects of Simulated Altitude on Maximal Oxygen Uptake and Inspiratory Fitness. Int J Exerc Sci, 10(1), 127-136.

Borges, T. O., Dascombe, B., Bullock, N., \& Coutts, A. J. (2015). Physiological characteristics of welltrained junior sprint kayak athletes. Int J Sports Physiol Perform, 10(5), 593-599. doi:10.1123/ijspp.2014-0292

Czuba, M., Fidos-Czuba, O., Płoszczyca, K., Zając, A., \& Langfort, J. (2018). Comparison of the effect of intermittent hypoxic training vs. the live high, train low strategy on aerobic capacity and sports performance in cyclists in normoxia. Biol Sport, 35(1), 39-48. doi:10.5114/biolsport.2018.70750

Czuba, M., Waskiewicz, Z., Zajac, A., Poprzecki, S., Cholewa, J., \& Roczniok, R. (2011). The effects of intermittent hypoxic training on aerobic capacity and endurance performance in cyclists. $J$ Sports Sci Med, 10(1), 175-183.

George, J. D., Vehrs, P. R., Allsen, P. E., Fellingham, G. W., \& Fisher, A. G. (1993). VO2max estimation from a submaximal 1-mile track jog for fit college-age individuals. Med Sci Sports Exerc, 25(3), 401-406. 
González, A. J., Hernández, D., De Vera, A., Barrios, Y., Salido, E., Torres, A., \& Terrados, N. (2006). ACE gene polymorphism and erythropoietin in endurance athletes at moderate altitude. Med Sci Sports Exerc, 38(4), 688-693. doi:10.1249/01.mss.0000210187.62672.b3

Granados, J., Gillum, T. L., Castillo, W., Christmas, K. M., \& Kuennen, M. R. (2016). "Functional" Respiratory Muscle Training During Endurance Exercise Causes Modest Hypoxemia but Overall is Well Tolerated. The Journal of Strength \& Conditioning Research, 30(3), 755-762. doi:10.1519/jsc.0000000000001151

Jagim, A. R., Dominy, T. A., Camic, C. L., Wright, G., Doberstein, S., Jones, M. T., \& Oliver, J. M. (2018). Acute Effects of the Elevation Training Mask on Strength Performance in Recreational Weight lifters. J Strength Cond Res, 32(2), 482-489. doi:10.1519/jsc.0000000000002308

Ke, Q., \& Costa, M. (2006). Hypoxia-inducible factor-1 (HIF-1). Mol Pharmacol, 70(5), 1469-1480. doi:10.1124/mol.106.027029

Kerr, R., Spinks, W., Leicht, A., Sinclair, W., \& Woodside, L. (2008). Physiological responses to 1000-m ergometer time-trial performance in outrigger canoeing. Journal of Sports Sciences, 26(11), 1219 1223. doi:10.1080/02640410802056484

Kido, S., Nakajima, Y., Miyasaka, T., Maeda, Y., Tanaka, T., Yu, W., ... Takayanagi, K. (2013). Effects of combined training with breathing resistance and sustained physical exertion to improve endurance capacity and respiratory muscle function in healthy young adults. J Phys Ther Sci, 25(5), 605-610. doi:10.1589/jpts.25.605

Lee, J. W., Bae, S. H., Jeong, J. W., Kim, S. H., \& Kim, K. W. (2004). Hypoxia-inducible factor (HIF-1)alpha: its protein stability and biological functions. Exp Mol Med, 36(1), 1-12. doi:10.1038/emm.2004.1

Mackenzie, R. W., Watt, P. W., \& Maxwell, N. S. (2008). Acute normobaric hypoxia stimulates erythropoietin release. High Alt Med Biol, 9(1), 28-37. doi:10.1089/ham.2007.1043

Markov, G., Spengler, C. M., Knöpfli-Lenzin, C., Stuessi, C., \& Boutellier, U. (2001). Respiratory muscle training increases cycling endurance without affecting cardiovascular responses to exercise. Eur J Appl Physiol, 85(3-4), 233-239. doi:10.1007/s004210100450

Park, H.-Y., \& Lim, K. (2017). Effects of Hypoxic Training versus Normoxic Training on Exercise Performance in Competitive Swimmers. J Sports Sci Med, 16(4), 480-488.

Porcari, J. P., Probst, L., Forrester, K., Doberstein, S., Foster, C., Cress, M. L., \& Schmidt, K. (2016). Effect of Wearing the Elevation Training Mask on Aerobic Capacity, Lung Function, and Hematological Variables. J Sports Sci Med, 15(2), 379-386.

Rocha, F. P. S., Louro, H., Matias, R., Brito, J., \& Costa, A. M. (2016). Determination of Aerobic Power Through a Specific Test for Taekwondo - A Predictive Equation Model. Journal of human kinetics, 53(1), 117-126. doi:doi:10.1515/hukin-2016-0016

Romer, L. M., McConnell, A. K., \& Jones, D. A. (2002). Inspiratory muscle fatigue in trained cyclists: effects of inspiratory muscle training. Med Sci Sports Exerc, 34(5), 785-792. doi:10.1097/00005768-200205000-00010

Wahl, P., Schmidt, A., Demarees, M., Achtzehn, S., Bloch, W., \& Mester, J. (2013). Responses of angiogenic growth factors to exercise, to hypoxia and to exercise under hypoxic conditions. Int J Sports Med, 34(2), 95-100. doi:10.1055/s-0032-1314815

Wiśniewska, A., Płoszczyca, K., \& Czuba, M. (2020). Changes in erythropoietin and vascular endothelial growth factor following the use of different altitude training concepts. J Sports Med Phys Fitness, 60(5), 677-684. doi:10.23736/s0022-4707.20.10404-3 
Tables

Table 1. The effects of the three intervention on 1000-m kayaking record, EPO, VEGF, lactate, and VO2max.

\begin{tabular}{|c|c|c|c|c|c|c|c|c|c|}
\hline \multirow{2}{*}{ Parameters } & \multicolumn{3}{|c|}{ Mask Group $(\mathbf{n}=10)$} & \multicolumn{3}{|c|}{ No Mask Group $(\mathbf{n}=\mathbf{1 0})$} & \multicolumn{3}{|c|}{ Control group $(n=10)$} \\
\hline & Pre & Post & $\begin{array}{l}\mathbf{p} \\
\text { Value }\end{array}$ & Pre & Post & $\begin{array}{l}\mathbf{p} \\
\text { Value }\end{array}$ & Pre & Post & p Value \\
\hline $\begin{array}{l}\text { 1000-m } \\
\text { kayaking } \\
\text { record } \\
\text { (seconds) }\end{array}$ & $157.1 \pm 4.0$ & $149.9 \pm 4.0$ & $<0.001$ & $156.6 \pm 6.7$ & $155.7 \pm 5.1 * *$ & 0.095 & $156.3 \pm 5.0$ & $155.4 \pm 155.4 *$ & 0.06 \\
\hline $\begin{array}{l}\mathbf{E P O} \\
(\mathrm{mU} / \mathrm{ml})\end{array}$ & $7.3 \pm 4.6$ & $7.35 \pm 3.6$ & 0.595 & $6.5 \pm 1.7$ & $5.9 \pm 2.1$ & 0.091 & $5.4 \pm 1.0$ & $5.3 \pm 1.4$ & 0.920 \\
\hline $\begin{array}{l}\text { VEGF } \\
(\mathrm{pg} / \mathrm{ml})\end{array}$ & $121.2 \pm 28.5$ & $135.5 \pm 14.9$ & 0.040 & $114.5 \pm 20.9$ & $132.4 \pm 8.9$ & 0.014 & $126.5 \pm 15.1$ & $134.2 \pm 8.7$ & 0.084 \\
\hline Lactate & $9.2 \pm 1.4$ & $8.0 \pm 2.1$ & 0.025 & $9.7 \pm 3.6$ & $7.5 \pm 1.2$ & 0.111 & $7.7 \pm 1.4$ & $9.6 \pm 2.1$ & 0.059 \\
\hline $\begin{array}{l}\text { VO2max } \\
\left(\mathrm{mL} \cdot \mathrm{kg}_{-}{ }^{1} \cdot \mathrm{min}^{-1}\right)\end{array}$ & $46.3 \pm 8.7$ & $48.6 \pm 5.2$ & 0.180 & $41.3 \pm 7.9$ & $47.6 \pm 10.0$ & 0.021 & $41.8 \pm 4.4$ & $45.1 \pm 8.0$ & 0.128 \\
\hline
\end{tabular}

